Arthroskopie 2009 · 22:265-266

DOI 10.1007/s00142-009-0516-0

Online publiziert: 17. September 2009

(c) Springer Medizin Verlag 2009

\author{
M. Dienst \\ OCM Orthopädische Chirurgie München
}

\title{
Aktuelle Trends und Grenzen der Hüftarthroskopie
}

häufig zu einer progredienten Sekundärarthrose. Die Literaturanalyse zeigt Rezidive nach arthroskopischem und offenem Vorgehen, sowohl ohne als auch nach subtotalen Synovektomien. Matthias Kusma und Mitarbeiter aus Homburg/Saar beschreiben charakteristische Befunde, die den Verdacht auf eine synoviale Erkrankung lenken sollten. Vor- und Nachteile des arthroskopischen und offenen Vorgehens bei Synovitiden wie der Chondromatose oder pigmentierten villonodulären Synovitis werden diskutiert.

Neben einem knöchernen Impingement ist kürzlich ein Weichteilimpingement zwischen Schleimhautfalten des Femurkopf-Hals-Übergangs und der Gelenkkapsel vorgestellt worden. Thierry Boyer und Mitarbeiter aus Paris beschäftigen sich als Rheumatologen bereits seit mehr als 20 Jahren mit der Arthroskopie des synovitischen Hüftgelenks. Das pektineofoveale Impingement zwischen medialer Schleimhautfalte und Gelenkkapsel wurde von ihnen erstbeschrieben.

Die Funktion des Ligamentum capitis femoris ist weiterhin nicht vollständig geklärt, diskutiert werden eine Bedeutung zur intrauterinen Blutversorgung des Femurkopfes und seine Funktion als Stabilisator und Rezeptororgan. Christoph Lampert aus St. Gallen geht im Detail auf die verschiedenen anatomischen und biomechanischen Aspekte ein, bevor pathologische Veränderungen des Ligaments und der Fossa acetabuli und die arthroskopische Evaluation und Therapie beschrieben werden.

Insbesondere bei der Behandlung des femoroazetabulären Impingements und der Hüftdysplasie spielt das Labrum acetabulare eine zentrale Rolle. Die Entscheidung über den Erhalt oder die Refixation des Labrum ist hier, über den hohen technischen Anspruch hinaus, auch für den erfahrenen Hüftarthroskopeur gelegentlich schwierig. Die Arbeitsgruppe um Hassan Sadri aus Genf/Riaz stellt ihren Behandlungsalgorithmus in Abhängigkeit von Verletzung und zugrundeliegender Erkrankung, Gewebedegeneration und Risstyp, vor.

Rekonstruktive arthroskopische Operationen am Hüftgelenk sind bislang nur in Ausnahmefällen indiziert. Mögliche Indikationen bestehen nach Verlust des Labrum acetabulare oder großen Knorpeldefekten, die nicht mittels klassischer Verfahren wie Abrasionsarthroplastik und Mikrofrakturierung behandelt werden können. Marc Philippon aus Vail/U.S.A. bewegt sich technisch im Hüftgelenk seit einigen Jahren auf höchstem Niveau. Zusammen mit seinen Mitarbeitern stellt er seine Erfahrung mit der Rekonstruktion des Labrum acetabulare mit einem Fascialata-Streifen vor.

Keine andere Erkrankung hat die gelenkerhaltende Hüftchirurgie der letzten 10 Jahre so stark geprägt wie das femoroazetabuläre Impingement. Während die operative Behandlung in den ersten Jahren ausschließlich über eine chirurgische Luxation erfolgte, haben sich seit 2003 arthroskopische Techniken entwickelt. Ich beschreibe Vor- und Nachteile der offenen und arthroskopischen Operationstechnik und stelle meinen aktuellen Behandlungsalgorithmus in Abhängigkeit von Impingement-Typ und -Schweregrad vor.
Synoviale Erkrankungen der Hüfte werden nicht selten übersehen, führen jedoch 
Die Indikationsliste für die Arthroskopie des Hüftgelenks wurde auf Komplikationen nach endoprothetischem Hüftersatz erweitert. Einsatzmöglichkeiten bieten sich bei unklaren Schmerzen, Impingement und Psoasirritationen. Die Arbeitsgruppe um Richard Villar aus London beschreibt ihre Erfahrungen nach Arthroskopien von Hüftgelenken, die mittels Oberflächenersatzprothesen endoprothetisch versorgt wurden.

Ich hoffe, dass wir mit dem vorliegenden Heft Ihr Interesse an dem spannenden Gebiet der arthroskopischen Hüftchirurgie wecken und dem bereits aktiven Hüftarthroskopeur den einen oder anderen operativen oder klinisch relevanten Tipp geben können.

Ihr

Michael Dienst

\section{Korrespondenzadresse}

\section{PD Dr. M. Dienst}

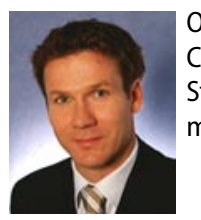

\section{G.-C. Zinn, E. Tabori, P. Weidenfeller Ambulantes Operieren - Praktische Hygiene}

Kissing: Verlag für Medizinische Praxis 2006, 1. Auflage, 259 S., (ISBN 3-938999-02-), Hardcover-Bindung, 89.00 EUR

Ambulante Operationen haben in den letzten Jahren enorm zugenommen. Auf Grund der in nächster Zeit zu erwartenden oder bereits eingetretenen Veränderungen des Gesundheitswesens mit der Etablierung von medizinischen Versorgungszentren und der Einführung der DRG-Abrechnung in den Krankenhäusern werden kurzzeitige, wenig risikobelastete Eingriffe im ambulanten Bereich weiter zunehmen. Mit dem vorliegenden Buch steht niedergelassenen Operateuren erstmals eine umfassende Einführung in dieses Gebiet zur Verfügung. Bei den Autoren handelt es sich um langjährig erfahrende Fachärzte für Hygiene und Umweltmedizin, zwei von den Autoren führen zusätzlich klinische Facharztbezeichnungen und haben entsprechende klinische Erfahrungen. Das Buch ist daher an keiner Stelle theoretisch oder abgehoben, sondern orientiert sich immer an den Bedürfnissen der Praxis. Die Gliederung des Buches erfolgt in rechtliche Grundlagen der Hygiene, bauliche Voraussetzungen und Bauplanung, Hygiene im Praxisbereich, Hygiene im OP, Hygiene bei der Anästhesie, Medizinprodukteaufbereitung, Infektionserfassung beim ambulanten Operieren und hygienisch-mikrobiologische Kontrollen. Zusätzliche Beiträge gehen auf spezielle Hygienemaßnahmen in bestimmten Fachgebieten wie z. B. der Ophthalmochirurgie ein. In Form einer CD-Rom sind dem Buch die aktuellen Empfehlungen des Robert-KochInstituts zum ambulanten Operieren sowie ein Rahmenhygieneplan und einschlägige berufsgenossenschaftliche Vorschriften beigefügt.

Ein Kernbeitrag ist derjenige über die Hygiene im OP, in dem zunächst die Einflussfaktoren auf das Wundinfektionsrisiko und anschließend die einzuhaltenden Hygienemaßnahmen dargestellt werden. Wichtig ist hier der Hinweis, dass OP-Masken nur noch während einer Operation im OP-Saal getragen werden müssen, während in den
Nebenräumen und auf dem Flur keine Masken getragen zu werden brauchen. Sehr nützlich sind auch die Hinweise für das anästhesiologische Management. Beispielsweise können heute Narkoseschläuche, wenn patientennahe Bakterienfilter verwendet werden, für mehrere Operationen eines Tages verwendet werden und brauchen erst am Ende des Arbeitstages ausgetauscht zu werden.

Das Buch gibt auch sehr gute Hinweise zur Strukturierung von Hygieneplänen und zu den routinemäßig durchzuführenden hygienischen Untersuchungen. Die Autoren empfehlen beispielsweise bei Sterilisationsgeräten nach wie vor noch die halbjährliche Prüfung mit den sogenannten "Sporenpäckchen". Dies erscheint auch durchaus vernünftig und praxisnah, zumal sich die kostenträchtigen Validierungen in ambulanten Bereichen noch nicht allgemein durchgesetzt haben. Für Desinfektionswaschmaschinen empfehlen die Autoren die halbjährliche Prüfung mit artifiziell kontaminierten Bioindikatoren. Auch dies scheint neben den technischen Wartungen unbedingt sinnvoll. Insgesamt ein sehr gelungenes Buch mit vielen praktischen und nützlichen Hinweisen für operativ tätige, niedergelassene Ärzte. Der Preis von 89,00 € incl. MwSt. zzgl. Versand erscheint für dieses schön ausgestattete Buch einschl. der CD-ROM gerechtfertigt.

M. Trautmann, Stuttgart 